\title{
Helianthemum marifolium subsp. molle (Cistaceae) en la provincia de Cuenca (Castilla-La Mancha, España)
}

\author{
O. GARCÍA CARDO ${ }^{1} \&$ J. M. MARTÍNEZ LABARGA²
}

\author{
${ }^{1}$ Empresa Pública de Gestión Ambiental de Castilla-La Mancha (GEACAM), ES-16004 Cuenca, España \\ ${ }^{2}$ Unidad docente de Botánica, Escuela Técnica Superior de Ingeniería de Montes, Forestal y del Medio Natural, \\ Departamento de Sistemas y Recursos Naturales, Universidad Politécnica de Madrid, ES-28040 Madrid, España
}

ORCID iD. O. GARCÍA CARDO: https://orcid.org/0000-0002-5515-9348,

J. M. MARTÍNEZ LABARGA: https://orcid.org/0000-0003-1565-7454

Autor para correspondencia: J. M. Martínez Labarga (juanmanuel.martinez@upm.es)

Editor: R. Vilatersana

Recibido 14 marzo 2019; aceptado 21 noviembre 2019; publicado on line 20 marzo 2020

\begin{abstract}
Helianthemum marifolium subsp. molle (Cistaceae) in the province of Cuenca (Castilla-La Mancha, Spain). - The presence of this endemic taxon from the east of the Iberian Peninsula in the province of Cuenca and in Castilla-La Mancha is reported, of which there no previous references were duly documented. In addition, notes are given on its taxonomic position and its habitat is commented. Finally, its degree of threat in the territory is shown, according to the IUCN criteria.
\end{abstract}

Key words: Altos de Cabrejas; chorology; conservation; flora; Iberian Peninsula.

\section{Resumen}

Helianthemum marifolium subsp. molle (Cistaceae) en la provincia de Cuenca (Castilla-La Mancha, España). - Se da a conocer la presencia de este taxón endémico del este de la península ibérica en la provincia de Cuenca y en Castilla-La Mancha, del cual no había referencias previas debidamente documentadas. Además se dan apuntes sobre su posición taxonómica y se comenta su hábitat. Por último, se muestra su grado de amenaza en el territorio, según los criterios de la UICN.

Palabras clave: Altos de Cabrejas; conservación; corología; flora; península ibérica.

\section{Cómo citar este artículo / Citation}

García Cardo, O. \& Martínez Labarga, J. M. 2020. Helianthemum marifolium subsp. molle (Cistaceae) en la provincia de Cuenca (Castilla-La Mancha, España). Collectanea Botanica 39: e003. https://doi.org/10.3989/collectbot.2020. v39.003

\section{Copyright}

(C) 2020 CSIC. This is an open-access article distributed under the terms of the Creative Commons Attribution 4.0 International (CC BY 4.0) License. 


\section{INTRODUCCIÓN}

Helianthemum marifolium (L.) Mill. subsp. molle (Cav.) G. López, descrito por Cavanilles como Cistus mollis Cav. de Peñagolosa (Cavanilles, 1796; Mateo \& Laguna, 2004), se extiende por el nordeste ibérico (Fig. 1A), desde el sur de Cataluña hasta el norte de la Comunidad Valenciana y montañas orientales de Teruel, en las provincias de Castellón, Tarragona y Teruel (López González, 1993: 407); también se ha documentado para la provincia de Valencia [Laguna et al., 1998: 164; H. marifolium subsp. molle in GBIF, 2018; Helianthemum origanifolium subsp. molle (Cav.) Font Quer \& Rothm. in GBIF, 2018], aunque dichas localidades se han puesto en duda (Mateo, 1999: 30).

\section{MATERIAL Y MÉTODOS}

Para contrastar la información taxonómica y corológica, se han consultado para la provincia de Cuenca los datos de los herbarios ABH, JACA, MA, MACB, MAF y VAL y los datos disponibles online (SIVIM, 2013; TPL, 2013; Anthos, 2018; Biodiversidad Virtual, 2018; GBIF, 2018; RJBColecciones, 2018).

\section{RESULTADOS}

Helianthemum marifolium (L.) Mill. Gard. Dict. ed. 8, n. 24 (1768) subsp. molle (Cav.) G. López in Anales Jard. Bot. Madrid 50(1): 55 (1992).

= Cistus mollis Cav., Icon. 3: 32, tab. 262 fig. 2 (1795-96) [basión.]; H. molle (Cav.) Pers., Syn. Pl. 2: 76 (1806); H. origanifolium subsp. molle (Cav.) Font Quer \& Rothm. in Cavanillesia 6: 161 (1934).

España, Cuenca: Fuentenava de Jábaga, Navalón, El Escalón, 30TWK5838, 1160 m, encinares sobre conglomerados, 08.XII.2016, O. García Cardo (v.v.) \& A. Ayllón; Fuentenava de Jábaga, Navalón, la Cuerda, 30TWK5937, 1160 m, encinar con pino negral sobre calizas, 04.V.2017, $O$. García Cardo 2420; Fuentenava de Jábaga-Villar del Saz de Navalón, cuestas vertientes al río Mayor de Navalón, 30TWK5543, $1050 \mathrm{~m}$, orientación E, en mosaico de pinar de pino carrasco repoblado, encinar y tomillar-aliagar sobre margas rojizas, 17.VI.2004, J. M. Martínez Labarga
94/2004-06-17 (MA 925009) (Martínez Labarga, 2014: 559); Fuentenava de Jábaga-Villar del Saz de Navalón, la Calzada, 30TWK5641, 1145 m, encinares sobre conglomerados, calizas, arenas y arcillas, 06.XII.2016, O. García Cardo (v.v.) \& J. M. García; Fuentenava de Jábaga-Villar del Saz de Navalón, la Calzada, 30TWK5641, 1140 m, encinares sobre suelos calcáreo, 15.IV.2017, O. García Cardo 2415 (MA 925726); Fuentenava de JábagaVillar del Saz de Navalón, laderas de Peña Canaleja sobre la Cañada Villar, 30TWK5743, 1100 m, orientación $\mathrm{W}$, en encinar-pinar de pino negral sobre areniscas calizas, 18.IX.2004, J. M. Martínez Labarga 18/2004-09-18.

Las localidades estudiadas se encuentran en 5 cuadrículas de UTM de $1 \mathrm{~km}$ de lado que se distribuyen en 2 cuadrículas de UTM de $10 \mathrm{~km}$ de lado (Fig. 1B), al norte de los Altos de Cabrejas, en el límite entre las comarcas de la Serranía Baja y La Alcarria. El conteo poblacional no se ha realizado, pero se trata de una planta que no es rara en las localidades visitadas.

\section{DISCUSIÓN}

Helianthemum marifolium subsp. molle es una pequeña matilla fruticulosa con ramas difusas o decumbentes; tallos floríferos ascendentes con abundantes pelos setosos fasciculados y patentes; hojas ovadas, orbiculares o cordiformes, con la nerviación marcada, uniformemente vellosas por haz y envés, más o menos concoloras, con los pelos del envés estrellados, largos y de radios erectos; hojas superiores generalmente no estipuladas; inflorescencia simple o con una rama basal, de (3)5-12 flores (Fig. 2).

Se encuadra en el subgénero Plectolobum Willk., Icon. Descr. P1. 2: 139 (1862), en la sección Pseudocistus Dunal in DC, Prodr. 1: 275 (1824) (López González, 1992; Aparicio et al., 2017). En este grupo se encuadran plantas con caracteres morfológicos bastante similares que dificultan muchas veces la adscripción correcta de las especies y aún más en el rango subespecífico. Entre las plantas ibéricas de la sección se encuentran los taxones pertenecientes a Helianthemum cinereum (Cav.) Pers., H. oelandicum (L.) Dum. Cours., H. pannosum Boiss., $H$. viscidulum Boiss. y a H. marifolium. Todos ellos tienen en común el ser plantas sufruticosas, más o 

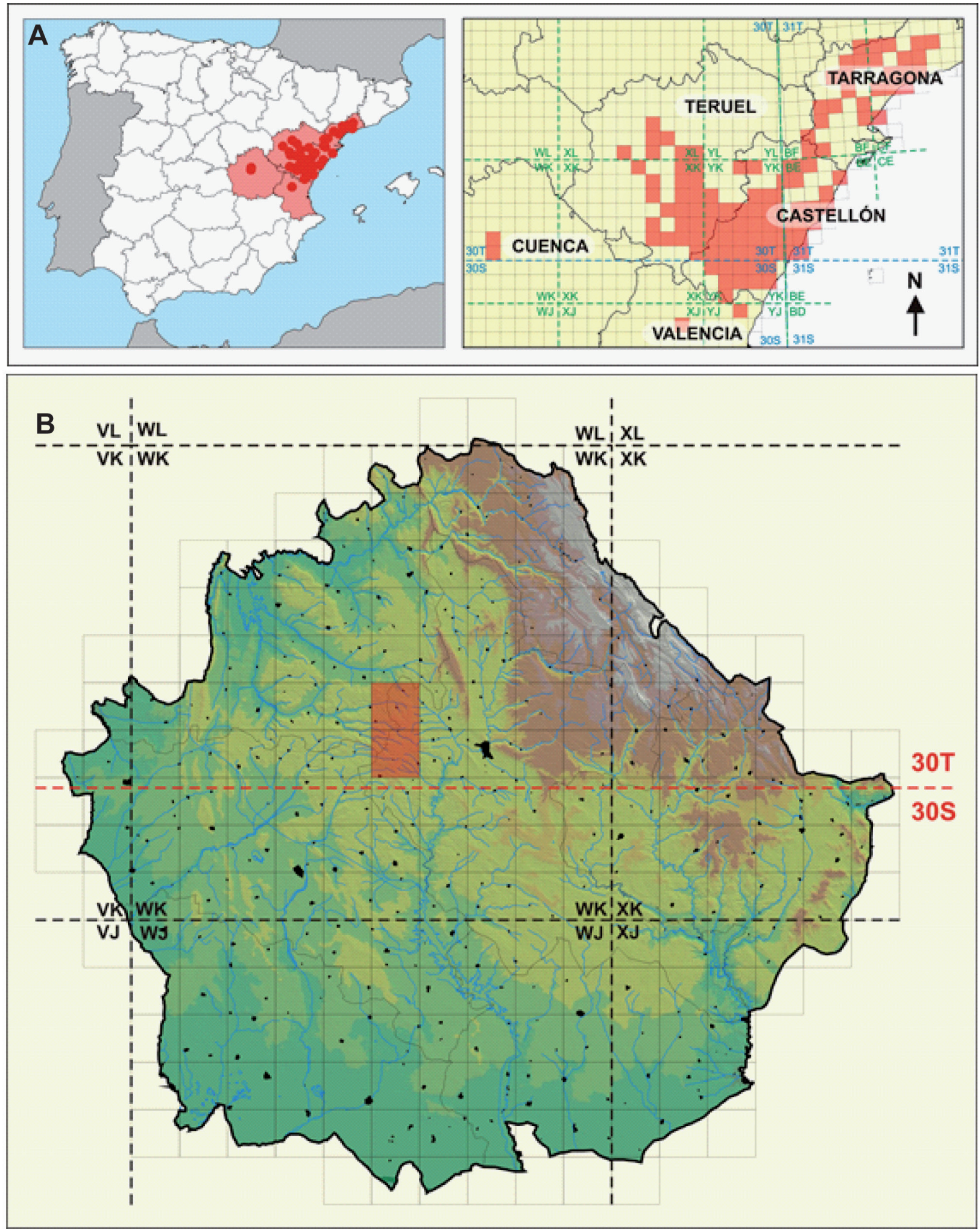

Figura 1. (A), distribución de Helianthemum marifolium subsp. molle en la península ibérica (fuente elaboración propia, a partir de http://colecciones.rjb.csic.es/, http://biodiver.bio.ub.es/biocat/, http://floragon.ipe.csic.es/, http://www.bdb.gva.es/ ca); (B), distribución de Helianthemum marifolium subsp. molle en cuadrículas UTM de $10 \mathrm{~km}$ en la provincia de Cuenca. 


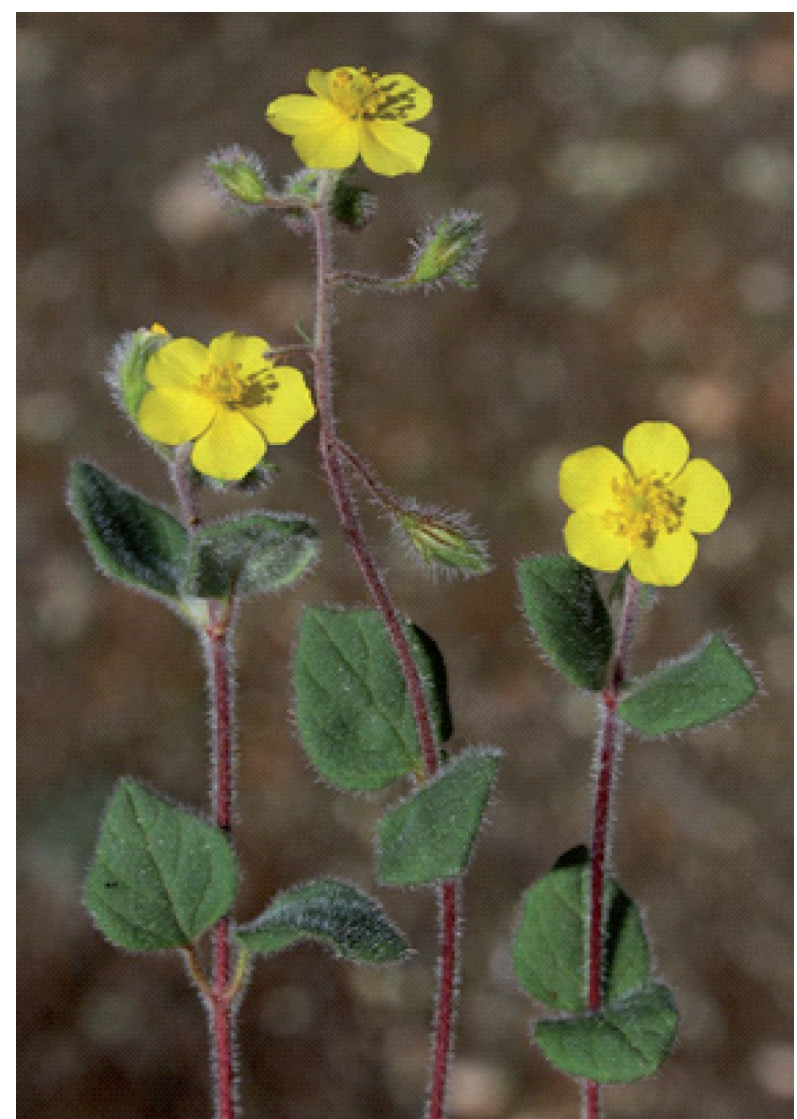

Figura 2. Imagen de Helianthemum marifolium subsp. molle en "La Calzada" (15.IV.2017; fotografía: O. García Cardo).

menos leñosas, con inflorescencias que nacen de brotes en roseta y de flores amarillas. (López González, 1993: 398).

Un criterio analítico en el grupo (Cavanilles, 1796; Mateo \& Arán, 1996; Mateo et al., 2013) separaría la planta aquí estudiada en la categoría específica con el nombre de $H$. molle, hecho que no resulta desacertado teniendo en cuenta que mantiene unos caracteres morfológicos bastante uniformes, como las hojas grandes, concoloras, vellosas y con la nerviación marcada. Y que los recientes estudios filogenéticos (Aparicio et al., 2017; Martín-Hernanz et al., 2017) hacen preciso profundizar en la reevaluación taxonómica de la sect. Pseudocistus y distinguen en cierta medida los taxones actualmente encuadrados en $H$. marifolium.

Caso aparte es la inclusión de estas plantas en H. origanifolium (Lam.) Pers. (Font Quer \& Rothmaler, 1934: 161) que podría estar muy justificado por la similitud morfológica de ambos taxones, aunque se diferencian perfectamente en las formas extremas por la forma y consistencia de las hojas y por el tipo de indumento, pero que no nos parece conveniente ya que $H$. molle se mantiene bastante estable en todas sus poblaciones, mientras que H. origanifolium, con un área de distribución más amplia, es más variable y presenta introgresiones, además de con la planta aquí estudiada, sobre todo con H. marifolium, hecho que ha justificado el tratamiento propuesto en Flora iberica (López González, 1992, 1993). En cualquier caso se trata de especies muy próximas en las que son frecuentes los híbridos y taxones infraespecíficos descritos relacionados con $H$. marifolium, $H$. molle o $H$. origanifolium (Mateo, 2012, 2013, 2017; Pérez Dacosta \& Mateo, 2012; Crespo et al., 2016).

La vegetación en las localidades consideradas es variada, como consecuencia de las diferentes exposiciones y diversidad de sustratos que se observan en estos montes. Hay desde matorrales ralos, tipo tomillar, a matorrales arbolados, con encinar bastante puro (Junipero thuriferaeQuercetum rotundifoliae Rivas-Martínez 1987) (Rivas Martínez et al., 2001), hasta encinar con quejigar (Quercus faginea Lam.) y pinar de pino negral [Pinus nigra Aiton subsp. salzmannii (Dunal) Franco]. Destaca la abundancia de especies del género Juniperus L., así como la de Arctostaphylos uva-ursi (L.) Spreng. y la prevalencia de especies leñosas de matorral heliófilo asociadas a etapas preforestales como Genista scorpius (L.) DC., Hormatophylla lapeyrousiana (Jord.) P. Küpfer, Ononis fruticosa L., Lavandula latifolia Medik., Sideritis incana L., Aphyllanthes monspeliensis L., Carex halleriana Asso, o diferentes taxones del género Helianthemum Mill., como $H$. hirtum (L.) Mill., H. gr. apenninum (L.) Mill., $H$. cinereum subsp. rotundifolium (Dunal) Greuter o el híbrido de este último por H. molle. En definitiva se trata de diversos matorrales basófilos de la Alianza Sideritido incanae-Salvion lavandulifoliae (Rivas Goday \& Rivas-Martínez 1969) Izco \& A. Molina 1989 y la Subalianza Xero-Aphyllanthenion Rivas Goday \& Rivas-Martínez 1969 em. Izco \& A. Molina 1989 (Rivas Martínez et al., 2001), los cuales forman parte de los citados encinares mixtos con quejigo y pino negral. La litología es variada, desde calizas hasta conglomerados $\mathrm{y}$ arenas, y presentan cierta querencia por suelos bastante pedregosos e incluso roquedos; en estas localidades son frecuentes las areniscas calizas. 
En la provincia de Castellón está bien distribuida (Laguna et al., 1998: 164) y es frecuente, de hecho ha sido motivo de varias asociaciones fitosociológicas como Helianthemo mollis-Ulicetum parviflorae Stübing, Peris et Costa 1989 o Helianthemo mollis-Hypericetum ericoidis R. Roselló 1994 (Rivas Martínez et al., 2001), hecho que confirma la abundancia local y la amplia diversidad de ambientes bioclimáticos observados para esta planta (Mateo, 1999).

\section{ESTADO DE CONSERVACIÓN}

No se han localizado otras localidades conquenses de este taxón endémico de la península ibérica (SIVIM, 2013; Anthos, 2018; Biodiversidad Virtual, 2018; GBIF, 2018), que alcanza el centro de la provincia. Las poblaciones conquenses son las únicas conocidas hasta la fecha en toda Castilla-La Mancha y constituyen una interesante disyunción respecto a su núcleo principal en la provincia de Castellón y sus confluencias con Teruel y Tarragona, por lo que la aportación aquí mostrada supone una importante ampliación de área - las poblaciones más cercanas se sitúan en la provincia de Teruel en la Sierra de Javalambre a unos $100 \mathrm{~km}$ - y una notable inclusión para el catálogo florístico conquense.

Respecto al estatus de conservación de esta subespecie endémica, no se encuentra en el Libro Rojo de la flora vascular española de 2008 y 2010 (Bañares et al., 2008, 2010), por lo que no se considera amenazado en las poblaciones levantinas, aragonesas y catalanas en las que estaba citada. El análisis territorial detallado muestra que no está contemplada en el Libro Rojo de plantas endémicas y amenazadas de Cataluña (Sáez et al., 2010), ni en el Catálogo de especies amenazadas de Aragón (Alcántara et al., 2007), pero si se encuentra contemplado en el Libro de la flora endémica, rara o amenazada de la Comunidad Valenciana (Laguna et al., 1998) por ser un casi-endemismo valenciano pero sin considerarse amenazado (categoría LRlc), por lo que no se incluye en su normativa propia sobre especies protegidas de fauna y flora (DOGV, 2013).

Esta subespecie debe ser considerada como amenazada en Castilla-La Mancha, pues el Decreto 33/1998 (DOCM, 1998) por el que se crea el Catálogo regional de especies amenazadas promueve especialmente la inclusión de entre otras: "las especies que tienen en Castilla-La Mancha su límite de distribución, con pequeñas y frágiles poblaciones de carácter marginal o relíctico" (objetivos 3b); por otro lado la Ley 9/1999 de Conservación de la naturaleza de Castilla-La Mancha (DOCM, 1999) en su artículo 63 relativo a los Principios Generales, en su apartado 4 menciona textualmente que: "se dará prioridad a la conservación a las especies endémicas, así como a aquellas otras cuya área de distribución sea muy limitada o su población muy escasa, y a las migratorias", además en su artículo 75 en su apartado 1d menciona: que se pueden incluir en la categoría "De interés especial" (IE) —en base a las categorías administrativas vigentes en el catálogo regional (DOCM, 2001) - a aquellas especies que sin estar contempladas en ninguna de las categorías precedentes (En peligro de extinción y Vulnerables) sean merecedoras de una atención particular en función de su valor científico, ecológico, cultural o por su singularidad, tal y como es el caso que aquí se presenta.

Y si se siguen los criterios de UICN (2012a), la aplicación de los mismos haría que pudiese catalogarse como Vulnerable VU B1ab(iii)+2ab(iii); D2, en base a que tiene en Cuenca y Castilla-La Mancha una extensión de presencia y área de ocupación restringida y con pocas localidades, pero tendría que comprobarse que la población disminuye para poder afirmar que es Vulnerable (IUCN, 2012a: 22) y cumple con los criterios b de "disminución continua, observada, inferida o proyectada". Aunque si bien la aplicación estricta de los mismos criterios con carácter local o regional (UICN, 2012b) permitiría: o bien, mantener la categoría de Vulnerable, en base a la escala de aplicabilidad, debido a que está aislada geográficamente de poblaciones coespecíficas fuera de la región (UICN, 2012b: 3, 15), o bien, conduciría a reevaluarla [en el proceso de secuencia de pasos, considerando que pudiera haber inmigración de propágulos desde las otras regiones en las que se encuentra el taxón (UICN, 2012b: 15)], como Casi Amenzada NT $^{\circ}$ (el símbolo de grado "o" sirve para indicar cambio de categoría) (IUCN, 2012b: 22). Este último tratamiento se justificaría porque las poblaciones estudiadas se presupone que son estables (UICN, 2012a: 9) y en los últimos años las formaciones vegetales en las que se encuentra están en buen estado. Esto comportaría la rebaja de un punto en el grado de amenaza de H. marifolium subsp. molle respecto a las previsiones iniciales. 
Por último indicar que los encinares basófilos en los que habita se encuentran incluidos en la Directiva 92/43/CEE relativa a la conservación de los hábitats naturales y de la fauna y flora silvestres (Comunidad Europea, 1992) en su anexo I bajo el código 9340 (Bartolomé et al., 2005: 246).

\section{AGRADECIMIENTOS}

Agradecemos a G. López González las enseñanzas recibidas sobre el género Helianthemum y al personal del herbario MA, en especial a J. L. Fernández Alonso, C. Baranda, L. Medina y C. Noya las facilidades para poder consultar el herbario. Por último a los revisores y al editor que con sus sugerencias han ayudado a que este modesto escrito pueda ser de utilidad.

\section{REFERENCIAS BIBLIOGRÁFICAS}

Alcántara, M., Viñuales, E. \& Sánchez Salcedo, E. 2007. Catálogo de especies amenazadas en Aragón. Departamento de Medio Ambiente, Gobierno de Aragón, Zaragoza.

Anthos 2018. Sistema de información de las plantas de España. Consultado en octubre de 2018, en http://www.anthos.es

Aparicio, A., Martín-Hernanz, S., Parejo-Farnés, C. et al. 2017. Phylogenetic reconstruction of the genus Helianthemum (Cistaceae) using plastid and nuclear DNA-sequences: systematic and evolutionary inferences. Taxon 66: 868-885. https://doi.org/10.12705/664.5

Bañares, A., Blanca, G., Güemes, J., Moreno, J. C. \& Ortiz, S. (Eds.) 2008. Lista Roja 2008 de la flora vascular española. Ministerio de Medio Ambiente, y Medio Rural y Marino, Sociedad Española de Biología de la Conservación de Plantas, Madrid.

Bañares, A., Blanca, G., Güemes, J., Moreno, J. C. \& Ortiz, S. (Eds.) 2010. Atlas y Libro Rojo de la flora vascular amenazada de España. Adenda 2010. Ministerio de Medio Ambiente, y Medio Rural y Marino, Sociedad Española de Biología de la Conservación de Plantas, Madrid.

Bartolomé, C., Álvarez, J., Vaquero, J., Costa, M., Casermeiro, M. A., Giraldo, J. \& Zamora, J. 2005. Los tipos de hábitat de interés comunitario de España: guía básica. Ministerio de Medio Ambiente, Madrid.

Biodiversidad Virtual 2018. Herbarium. Consultado en octubre de 2018, en http://www.biodiversidadvirtual.org/herbarium

Cavanilles, A. J. 1796. Icones et descriptiones plantarum 3(2). Regia Typographia, Madrid.

Comunidad Europea 1992. Directiva 92/43/CEE del Consejo del 21 de mayo de 1992 relativa a la conservación de los hábitats naturales y de la fauna y flora silvestres. Diario Oficial de las Comunidades Europeas 206: 7-37.

Crespo, M. B., Alonso, M. A., Vicente, A. \& Villar, J. L. 2016. A new North African subspecies in the Helianthemum origanifolium aggregate $(H$. subg. Plectolobum, Cistaceae). Phytotaxa 252: 263-272. https://doi.org/10.11646/phytotaxa.252.4.2

DOCM [Diario Oficial de Castilla-La Mancha] 1998. Decreto 33/1998, de 5 de mayo de 1998 por el que se crea el Catálogo regional de especies amenazadas de Castilla-La Mancha. Diario Oficial de Castilla-La Mancha 22: 3391-3398.

DOCM [Diario Oficial de Castilla-La Mancha] 1999. Ley 9/1999, de 26 de mayo, de Conservación de la naturaleza. Diario Oficial de Castilla-La Mancha 40: 4066-4091.

DOCM [Diario Oficial de Castilla-La Mancha] 2001. Decreto 200/2001, de 6 de noviembre de 2001 por el que se modifica el Catálogo regional de especies amenazadas. Diario Oficial de Castilla-La Mancha 119: 12825-12827.

DOGV [Diario Oficial de la Generalitat Valenciana] 2013. Orden 6/2013, de 25 de marzo, de la Conselleria de Infraestructuras, Territorio y Medio Ambiente, por la que se modifican los listados valencianos de especies protegidas de flora y fauna [2013/3116]. Diario Oficial de la Generalitat Valenciana 6996: 8682-8690.

Font Quer, P. \& Rothmaler, W. 1934. Generum plantarum ibericarum revisio critica, I. Helianthemum Adans. Subgen. Plectolobum Willk. - Sectio Chamaecistus ej. Cavanillesia 6: $148-174$.

GBIF [Global Biodiversity Information Facility] 2018. Consultado en marzo de 2018, en www.gbif.org

Laguna, E., Crespo, M. B., Mateo, G. et al. 1998. Flora endémica rara o amenazada de la Comunidad Valenciana. Conselleria de Medi Ambient, Generalitat Valenciana, Valencia.

López González, G. 1992. Apuntes para justificar el tratamiento del género Helianthemum Miller, s.l. (Cistaceae), en Flora iberica. Anales del Jardín Botánico de Madrid 50: 35-63.

López González, G. 1993. Helianthemum Mill. In: Castroviejo, S., Aedo, C., Cirujano, S. et al. (Eds.), Flora iberica 3. Plumbaginaceae (partim)-Capparaceae. Real Jardín Botánico, CSIC, Madrid: 365-421.

Martín-Hernanz, S., Albaladejo, R. G., Rubio, E. \& Aparicio, A. 2017. Alta resolución filogenética en el género Helianthemum mediante el empleo de marcadores moleculares derivados de las técnicas NGS. Comunicación oral presentada en el VIII Congreso de Biología de la Conservación de Plantas (Madrid, España, 4-7 julio 2017).

Martínez Labarga, J. M. 2014. Estudios corológicos de plantas vasculares en la cuenca media del Tajo. Tesis Doctoral, Universidad Politécnica de Madrid, Madrid.

Mateo, G. 1999. Comentarios y adiciones al volumen $8^{\circ}$ del Atlas Corológico ORCA. Flora Montiberica 12: 29-32.

Mateo, G. 2012. Nuevos táxones del género Helianthemum Mill. en la zona oriental de la Península Ibérica, I. Flora Montiberica 50: 30-43.

Mateo, G. 2013. De flora valentina, XII. Flora Montiberica 55: 86-96

Mateo, G. 2017. Nuevos táxones del género Helianthemum Mill. Flora Montiberica 69: 58-66.

Mateo, G. \& Arán, V. J. 1996. Nuevos datos sobre la flora de la provincia de Cuenca, III. Flora Montiberica 3: 92-96.

Mateo, G., Crespo, M. B. \& Laguna, E. (Eds.) 2013. Flora valentina 2. Fundación de la Comunidad Valenciana para el Medio Ambiente, Valencia.

Mateo, G. \& Laguna, E. 2004. Especies de la flora valenciana descritos por Cavanilles. Flora Montiberica 28: 23-28.

Pérez Dacosta, J. M. \& Mateo, G. 2012. Nuevos táxones del género Helianthemum Mill. en la zona oriental de la Península Ibérica, II. Flora Montiberica 50: 44-61.

Rivas-Martínez, S., Fernández-González, F., Loidi, J., Lousã, M. \& Penas, A. 2001. Syntaxonomical checklist of vascular plant communities of Spain and Portugal to association level. Itinera Geobotanica 14: 5-341. 
RJB-Colecciones [Real Jardín Botánico-Colecciones] 2018. Herbario del Real Jardín Botánico. Consultado en octubre de 2018, en http://colecciones.rjb.csic.es/

Sáez, L., Aymerich, P. \& Blanché, C. 2010. Llibre Vermell de les plantes vasculars endèmiques $i$ amenaçades de Catalunya. Argania Editio, Barcelona.

SIVIM [Sistema de Información de la Vegetación Ibérica y Macaronésica] 2013. Consultado en octubre de 2018, en http://www.sivim.info/sivi/

TPL [The Plant List] 2013. The Plant List. A working list of all plant species v1.1. Consultado en octubre de 2018, en http://www.theplantlist.org/
UICN [Unión Internacional para la Conservación de la Naturaleza] 2012a. Categorías y criterios de la Lista Roja de la $U I C N$ v3.1. Segunda edición. UICN, Gland \& Cambridge.

UICN [Unión Internacional para la Conservación de la Naturaleza] 2012b. Directrices para el uso de los criterios de la Lista Roja de la UICN a nivel regional y nacional v4.0. UICN, Gland \& Cambridge. 